\title{
Numerical analysis of suction influence on the behaviour of unsaturated elasto-plastic soil medium
}

\author{
Pawet Fedczuk* \\ Opole University of Technology, Faculty of Civil Engineering and Architecture, Katowicka 48 Street, \\ 45-061 Opole, Poland
}

\begin{abstract}
The paper presents a concept of numerical analysis of suction influence on behaviour of partially saturated soil medium. Behaviour of soil is described by elasto-plastic model of unsaturated soil [1]. A numerical procedure of stress path simulation in the triaxial test [2] is used for the analysis of one non-standard shear path (for 3 suction levels).
\end{abstract}

\section{Introduction}

Applying complex constitutive relationships to describe the behaviour of unsaturated soils used in geotechnics requires verification of their ability to model correctly. It is implemented through a comparative analysis of the results of triaxial tests with the results of numerical simulation of the appropriate stress path. A simplifying assumption is usually made that at each point of the sample an identical state of stress and strain prevails, which reduces the task size to one material point. Proposals of this approach to the problem (for elasto-plastic models of saturated soils) can be found in publications [3, 4].

This paper presents a concept of a method of simulating stress path which takes into account influence of suction on the behaviour of a partially saturated soil medium. Selected stress path at the material point of soil, models the step-iterative computational technique [2] (using the modified concept of Newton-Raphson algorithm). The state of plasticity is specified by means of the corrected Nayak-Zienkiewicz method $[2,5]$. The presentation of the method is preceded by an introduction defining the basic relations of the models included in the extended theory of critical state $[1,6,7]$. Next, the concept of the computational procedure is discussed and its algorithm is given. The behaviour of unsaturated soil is described by the author's elasto-plastic model of such a medium [6, 1]. This method of analysis is illustrated by numerical simulation of stress path, realizing an equivalent of a nonstandard shear path in partially saturated kaolin for three different suction levels.

\footnotetext{
${ }^{*}$ Corresponding author: p.fedczuk@po.opole.pl
} 


\section{Theoretical basis}

\subsection{Effective stress, net stress and suction}

Stress state in any material point of partially saturated soil medium is characterized by component $\sigma_{i j}^{\prime}$ of effective stress vector $\boldsymbol{\sigma}^{\prime}$ defined by relationship $[8,1]$

$$
\sigma_{i j}^{\prime}=\left(\sigma_{i j}-\delta_{i j} u_{a}\right)+\delta_{i j} \chi\left(u_{a}-u_{w}\right)=\sigma_{i j}^{n}+\delta_{i j} \chi s \quad(i, j=1,2,3)
$$

in which: $\sigma_{i j}$ - is the component of total stress vector $\boldsymbol{\sigma}, \chi-$ is a parameter depending on saturation level of the soil (degree of saturation), $\delta_{i j}-$ is Kronecker's delta, $\sigma^{n}{ }_{i j}-$ is a component of the net stress vector $\boldsymbol{\sigma}^{n}, s$-suction (constituting the difference between gas pressure $u_{a}$ and water pressure $u_{w}$, existing in pores). Net equivalents of standard invariant effective values - mean stress $p^{\prime}$ and shear stress $q^{\prime}$ - are specified by the formulae

$$
\begin{gathered}
p^{n}=p-u_{a}=p^{\prime}-u_{a}-\chi s \\
q^{n}=q^{\prime}
\end{gathered}
$$

\subsection{Triaxial test}

The triaxial test of soil depends on shortening the cylindrical soil sample under the conditions of control of selected components of the stress state or strain state. In the case of experimental realization of the stress path, the assumed state of stress and suction is monitored, and the strain is observed. Usually results are reduced to a set of conjugate invariant values: effective shear stress $q^{\prime}$, net mean stress $p^{n}$, strain intensity $\varepsilon_{q}$, volume strain $\varepsilon_{v}$ and suction $s$. They are presented in the form of a set of correlated graphic characteristics.

\subsection{Modelling of behaviour of unsaturated soil}

Procedure of stress path simulation is intended for models that take into account a set of the following assumptions. Partially saturated soil medium is treated as isotropic. Soil pores are partially filled with water and gas (in the form of bubbles in water). Suction, being the effect of soil unsaturation, is an independent variable.

The elastic behaviour of partially saturated soil skeleton is defined by extension elasticity rule $[7,8]$

$$
d \boldsymbol{\sigma}^{n}=\mathbf{D}\left(d \boldsymbol{\varepsilon}^{e}-\mathbf{m}_{H} d s\right)
$$

in which: $d \boldsymbol{\sigma}^{n}-$ denotes vector of net stress increment, $\mathbf{D}-$ is constitutive matrix of soil elasticity (in drained conditions), $\mathbf{m}_{H}=\{0,3 / H\}^{T}$ - is the vector taking into account the effect of unsaturation of soil, $d \boldsymbol{\varepsilon}^{e}-$ is the vector of elastic part of strain increment $d \boldsymbol{\varepsilon}, d s-$ is the suction increment, $H$ - is modulus of unsaturated soil. However, the plastic behaviour is specified in the theory of critical state [9] in the extended form [7], taking into account the influence of suction on the stress state. This behaviour is modelled by the following pair of relationships: yield surface equation and hardening rule

$$
\begin{gathered}
F\left(p^{n}, q^{\prime}, s, \eta\right)=0 \\
\eta=\eta\left(\boldsymbol{\varepsilon}^{p}, s\right)
\end{gathered}
$$


where: $\eta$-denotes scalar hardening parameter, $\boldsymbol{\varepsilon}^{p}-$ is the plastic part of strain vector $\boldsymbol{\varepsilon}$.

\subsection{Incremental equation of elasto-plasticity}

Any increase of stress state and strain state (put in the form of relations $d \boldsymbol{\sigma}^{n}=\left\{d q^{\prime}, d p^{n}\right\}^{T}$, $\left.d \boldsymbol{\varepsilon}=\left\{d \varepsilon_{q}, d \varepsilon_{v}\right\}^{T}\right)$ at the material point of skeleton of unsaturated soil is connected with a set of 6 basic dependencies: constitutive rule of elasticity (3), yield surface equation (4.a), and rules: hardening (4.b), strain additive, plastic flow and consistency condition

$$
\begin{gathered}
d \boldsymbol{\varepsilon}=d \boldsymbol{\varepsilon}^{e}+d \boldsymbol{\varepsilon}^{p} \\
d \boldsymbol{\varepsilon}^{p}=d \lambda\left\{\frac{\partial F}{\partial \boldsymbol{\sigma}^{n}}\right\}=d \lambda \mathbf{a}_{F} \\
d F=\mathbf{a}_{F}^{T} d \boldsymbol{\sigma}^{n}+\frac{\partial F}{\partial s} d s+\frac{\partial F}{\partial \eta} d \eta=0
\end{gathered}
$$

Quantities $d \boldsymbol{\varepsilon}^{e}, d \boldsymbol{\varepsilon}^{e}$ denote increment vectors of elastic and plastic parts of strain increment $d \boldsymbol{\varepsilon}, d \lambda$ - is scalar multiplier, $\mathbf{a}_{F}-$ is gradient of plasticity function $F, \partial F / \partial s, \partial F / \partial \eta-$ are derivatives of yield function $F$ for suction $s$ and hardening parameter $\eta$.

The standard procedure described in [7], realized in a form analogical to classical [5], gives an incremental constitutive equation of elasto-plastic partially saturated soil medium

$$
\begin{gathered}
d \boldsymbol{\sigma}^{n}=\left[\mathbf{D}-\frac{\left(\mathbf{D} \mathbf{a}_{F}\right)\left(\mathbf{a}_{F}^{T} \mathbf{D}\right)}{\mathbf{a}_{F}^{T} \mathbf{D} \mathbf{a}_{F}+K_{F}}\right] d \boldsymbol{\varepsilon}-\left[\mathbf{D} \mathbf{m}_{H}+\frac{\left(\mathbf{D} \mathbf{a}_{F}\right)\left(\mathbf{a}_{F}^{T} \mathbf{D} \mathbf{m}_{H}\right)}{\mathbf{a}_{F}^{T} \mathbf{D} \mathbf{a}_{F}+K_{F}}-\right. \\
\left.\frac{\left(\mathbf{D} \mathbf{a}_{F}\right)\left(\frac{\partial F}{\partial s}+\frac{\partial F}{\partial \eta} \frac{\partial \eta}{\partial s}\right)}{\mathbf{a}_{F}^{T} \mathbf{D} \mathbf{a}_{F}+K_{F}}\right] d s=\left(\mathbf{D}-\mathbf{D}^{p}\right) d \boldsymbol{\varepsilon}-\left(\mathbf{D} \mathbf{m}_{H}-\mathbf{D}^{p s}\right) d s, \\
K_{F}=-\frac{\partial F}{\partial \eta}\left\{\frac{\partial \eta}{\partial \boldsymbol{\varepsilon}^{p}}\right\}^{T} \mathbf{a}_{F},
\end{gathered}
$$

where $K_{F}$ denotes the plastic hardening modulus, but $\mathbf{D}^{p}, \mathbf{D}^{p s}-$ constitutive matrix of soil elasticity and for suction.

\section{Numerical simulation of stress path}

\subsection{Concept of the procedure}

From a mathematical point of view, the considered problem is a nonlinear initial-boundary problem, requiring integration of the elasto-plastic constitutive equation (6) for the assumed boundary conditions. It is defined by a parametric function describing this path (shape and its characteristic points, usually initial and final) by means of a relation (consisting of a sequence of straight and nonlinear sections) specifying the increase in sample load correlated with the suction change $s$

$$
f\left(\boldsymbol{\sigma}^{n}, s\right)=\sum_{(i)} f_{(i)}\left(\boldsymbol{\sigma}^{n}, s\right)
$$


in which $f_{(i)}\left(\boldsymbol{\sigma}^{n}, s\right)$ - denotes the equation of the section $(i)$ of the stress trajectory.

The Newton-Raphson method is used for the solution, requiring the division of load and suction into a series of coupled increments and a cyclic solution of the equivalent differential elasto-plasticity equation. For such a defined relationship between finite stress increments $\Delta \boldsymbol{\sigma}^{n}$, suction $\Delta s$ and strain $\Delta \boldsymbol{\varepsilon}$, the initial-boundary condition is reduced to a pair of relations specifying the division of load and suction

$$
\begin{aligned}
\boldsymbol{\sigma}^{n} & =\sum \Delta \boldsymbol{\sigma}^{n} \\
s & =\sum \Delta s
\end{aligned}
$$

The course of the procedure is as follows. After applying another pair of stress increments $\Delta \boldsymbol{\sigma}^{n}$ and suction $\Delta s$, the elastic part of the strain increment $\Delta \boldsymbol{\varepsilon}^{e}$ from elastic rule (3) is calculated and the corresponding plastic part of the stress increment $\Delta \boldsymbol{\sigma}^{p}$ is determined using the modified procedure of Nayak-Zienkiewicz [5]. The finally obtained plastic parts of $\Delta \boldsymbol{\sigma}^{p}$ are treated as an elastic load in the cycle of internal iterations, repeated until the appropriate convergence is achieved.

\subsection{Algorithm of the calculation procedure}

The method of solution is a development of the concept given in [2], taking into account the suggestions included in the publications $[10,11]$. Implementation of the stress path in the unsaturated soil medium is modelled by the Newton-Raphson step-iterative technique. The operational algorithm of such a procedure comprises:

1) assumption of the initial parameters of soil model,

2) determination of constitutive matrix of soil elasticity $\mathbf{D}$, the vector taking into account the influence of unsaturation of soil $\mathbf{m}_{H}$ and plasticity function $F_{0}$ for initial state,

3) division of load $\boldsymbol{\sigma}^{n}$ and suction $s$ into a series (i) increments $\Delta \boldsymbol{\sigma}^{n(i)}$ and $\Delta s^{(i)}$,

4) realisation for $(i)$ incremental steps the cycle taking into account:

a) determination of the strain increment $\Delta \boldsymbol{\varepsilon}^{e(i)}$ from rule of elasticity

$$
\Delta \boldsymbol{\varepsilon}^{e(i)}=\mathbf{D}^{-1} \Delta \boldsymbol{\sigma}^{n(i)}+\mathbf{m}_{H} \Delta s^{(i)}
$$

b) establishing the plastic part of stress increment $\Delta \boldsymbol{\sigma}^{p(i)}$ with use of modified NayakZienkiewicz procedure [2],

c) checking the condition of interruption of the iterative cycle

$$
\sqrt{\left[\Delta \boldsymbol{\sigma}^{n(i)}\right]^{T} \Delta \boldsymbol{\sigma}^{n(i)}} \leq \tau \sqrt{\left[\Delta \boldsymbol{\sigma}_{(0)}^{n(i)}\right]^{T} \Delta \boldsymbol{\sigma}_{(0)}^{n(i)}}
$$

where: $\Delta \boldsymbol{\sigma}^{(i)}(0), \Delta \boldsymbol{\sigma}^{(i)}$ - are vectors of stress increments in the first and the next iterative step, $\tau$ - is the coefficient of convergence,

d) in the case of non-fulfilment of the condition (10) - a return to point (b) preceded by determining the deformation increment from dependence

$$
\Delta \boldsymbol{\varepsilon}^{e(i)}=\mathbf{D}^{-1} \Delta \boldsymbol{\sigma}^{p(i)}+\mathbf{m}_{H} \Delta s^{p(i)}
$$

e) in the case of fulfilment of the condition (10) - transition to point (5) (for the next incremental step) or ending of calculations (after executing $(i)$ incremental steps).

\section{Elasto-plastic model of the unsaturated soil}




\subsection{Yield surface}

The behaviour of soil describes author's elasto-plastic model of unsaturation soil medium $[6,1]$. Plastic properties of unsaturated soil medium are specified by the following pair of relationships: yield surface equation and hardening rule.

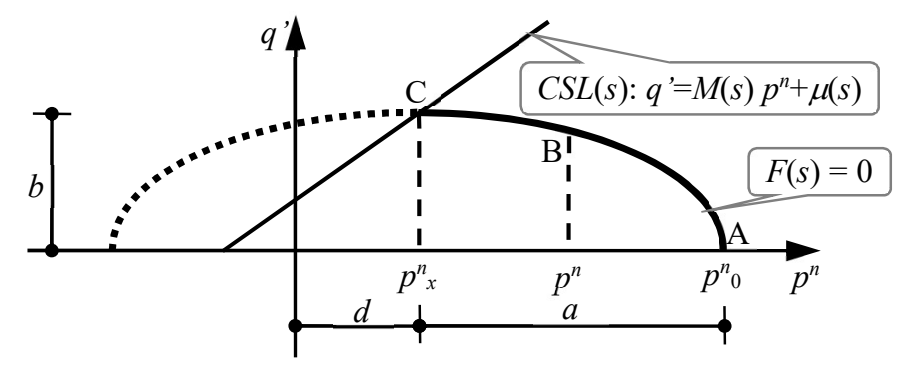

Fig. 1. Yield surface $F$.

Yield surface [6] is described by modified Wheeler and Sivakumar's equation [12]. This expression is based on ellipse equation (fig. 1) in canonical form (captured on the plane " $q$ ' $-p$ " ' for a specific level of suction $s$ )

$$
F\left(q^{\prime}, p^{n}, s\right)=\frac{\left(q^{\prime}\right)^{2}}{\left[M(s) \zeta p^{n}{ }_{0}+\mu(s)\right]^{2}}+\frac{\left(p^{n}-\zeta p^{n}\right)^{2}}{\left(p^{n}{ }_{0}-\zeta p_{0}\right)^{2}}-1=0
$$

A pair of model parameters $M(s)$ and $\mu(s)$ determine the position of momentary critical state line $\operatorname{CSL}(s)$ (current for suction level $s$ ). Relationship between characteristic net values of medium stress $p^{n}{ }_{x}$ i $p^{n}$ (defining the position of the vertex $\mathrm{C}$ and the beginning $\mathrm{A}$ of the ellipse respectively) is specified by this relation

$$
p^{n}{ }_{x}=\zeta p^{n}
$$

Elastic state defined by rule (3) rules inside the ellipse. This state is specified by three modules: shear $G$, bulk modulus $\mathrm{K}$ and modulus of unsaturated soil $H$.

\subsection{Hardening rule}

The hardening rule constitutes generalization of the classical concept for Modified Cam-Clay model [9]. It connects the plastic part of void ratio $\Delta e^{p}(s)$ with the state of net medium stress $p^{n}$. This rule is an effect of analysis of 2 sets of consolidation characteristics in system " $e-\ln$ $p^{n}$ " (fig. 2) for transition variant of stress state from level (1) to (2) (illustrated by the vector connecting points $\mathrm{B}_{1}$ and $\mathrm{B}_{2}$ ), corresponding with suction change $\Delta s=s_{2}-s_{1}$. The suction level (j) $(j=1,2)$ is characterized by a bundle of consolidation lines: normal $N C L$, limit $C S L$, indirect $I L$ and the elastic swelling $E L$, defined by dependencies

$$
\begin{gathered}
e=N\left(s_{j}\right)-1-\lambda\left(s_{j}\right) \ln p^{n} \\
e=\Gamma\left(s_{j}\right)-1-\psi\left(s_{j}\right) \ln p^{n} \\
e=N^{*}\left(s_{j}\right)-1-\lambda *\left(s_{j}\right) \ln p^{n} \\
e=e_{k j}-\kappa \ln p^{n}
\end{gathered}
$$


where: $N^{*}\left(s_{j}\right), \lambda^{*}\left(s_{j}\right)$ - denote a pair of coefficients of the equation of the indirect consolidation line $I L$ (the shift and slope respectively), while $N\left(s_{j}\right), \lambda\left(s_{j}\right)$, and $\Gamma\left(s_{j}\right), \psi\left(s_{j}\right)$ and $e_{k j}, \kappa$ - analogous pairs of coefficients for the lines $N C L, C L S$ and $E L$.

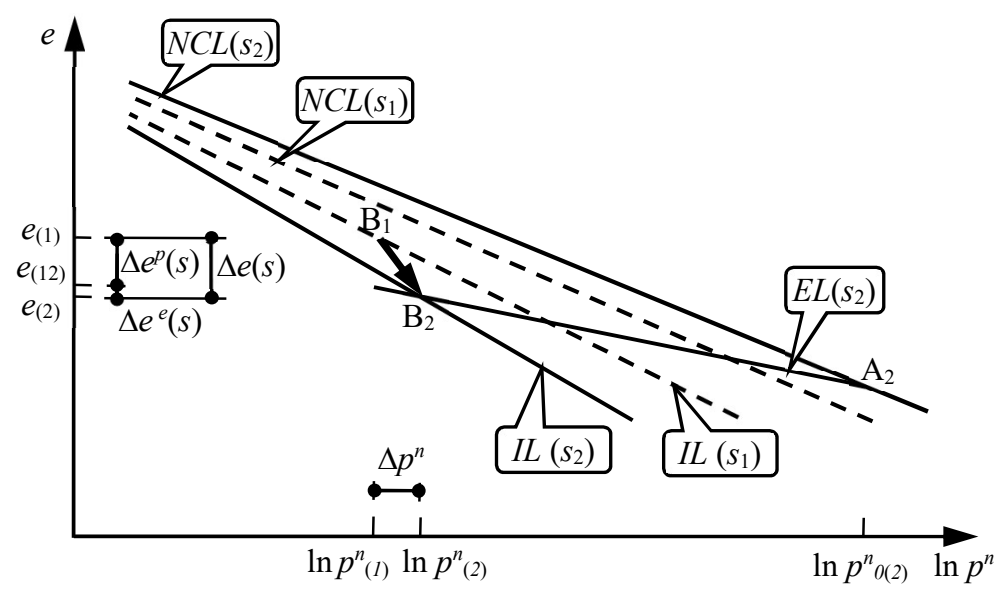

Fig. 2. Consolidation characteristics for transition from level (1) to level (2).

Elemental transformations [6], taking into account the additive rule, binding the total, elastic and plastic part of void ratio increment in form of relation $\Delta e^{p}(s)=\Delta e(s)-\Delta e^{e}(s)$, gives the final form of hardening rule

$$
\begin{gathered}
p^{n}{ }_{(2)}=\left\{\left(p^{n}{ }_{(1)}\right)^{\delta} \exp \left[\Delta e^{p}(s)-\alpha\right]\right\}^{1 / \beta} \\
\alpha=\left[N^{*}\left(s_{2}\right)-N^{*}\left(s_{1}\right)\right] \\
\delta=\left[\kappa-\lambda *\left(s_{1}\right)\right] \\
\beta=\left[\kappa-\lambda *\left(s_{2}\right)\right]
\end{gathered}
$$

This relationship is complemented by expression for net mean stress values $p^{n}$ and $p^{n}{ }_{x}$, defining correction of position yield surface $F$ in state (2) (i.e. coordinates of points $\mathrm{A}_{2}$ in system ' $q$ ' $-p^{n "}$ "). It is determined as the coordinate of the intersection point of the line $\operatorname{CSL}\left(s_{2}\right)$ and swelling line $E L\left(s_{2}\right)$ (passing through the point $\mathrm{B}_{2}\left(p^{n}(2), e_{(2)}\right)$ ), getting the following dependence as a result

$$
\begin{aligned}
& p^{n}{ }_{0(2)}=\exp \left\{\frac{\Gamma\left(s_{2}\right)-\xi}{\psi\left(s_{2}\right)-\kappa}\right\} \\
& \xi=1-e_{(2)}\left(s_{2}\right)+\kappa \ln p^{n}{ }_{(2)}
\end{aligned}
$$

\section{Example of stress path simulation}

\subsection{Analysis program}

The presented method of analysis is illustrated by the numerical simulation of the stress path, realizing the equivalent of a non-standard shear path in partially saturated kaolin. The behaviour of the soil medium describes the discussed elasto-plastic model of unsaturated soil 
medium $[6,1]$. A comprehensive specification of elastic constants is given in Table 1 , and the coordinates of the consolidation lines in Table 2.

Table 1. Elastic parameters of kaolin.

\begin{tabular}{|c|c|c|c|}
\hline$G[\mathrm{kPa}]$ & $K[\mathrm{kPa}]$ & $H[\mathrm{kPa}]$ & $\kappa$ \\
\hline 5200 & 15300 & 4680 & 0,02 \\
\hline
\end{tabular}

Calculations with using the program $3 \mathrm{OS}$ have been made for 3 variants of suction $s$ equal to $0 \mathrm{kPa}, 50 \mathrm{kPa}$ and $100 \mathrm{kPa}$ respectively. Computing process is characterized by additional conditions for the program: load division into $n=50$ identical stress increments $\Delta \sigma$ and fulfilment of convergence condition (4.c) for constant $\tau=10^{-8}$.

Table 2. Coordinates of the consolidation lines for kaolin [12].

\begin{tabular}{|c|c|c|c|c|c|c|c|}
\hline$j$ & $s_{j}[\mathrm{kPa}]$ & $M\left(s_{j}\right)$ & $\begin{array}{c}\mu\left(s_{j}\right) \\
{[\mathrm{kPa}]}\end{array}$ & $N\left(s_{j}\right)$ & $\lambda\left(s_{j}\right)$ & $\Gamma\left(s_{j}\right)$ & $\psi\left(s_{j}\right)$ \\
\hline 1 & 0 & 0,813 & 0,0 & 2,052 & 0,128 & 2,011 & 0,110 \\
\hline 2 & 50 & 0,873 & 27,1 & 2,087 & 0,155 & 1,998 & 0,109 \\
\hline 3 & 100 & 0,933 & 54,2 & 2,122 & 0,182 & 1,984 & 0,108 \\
\hline
\end{tabular}

\subsection{Analysis of results}

The results of the performed calculations are shown in fig. 3 in the form of three conjugated characteristics (stress " $q$ ' $-p^{n}$ " and strain " $\varepsilon_{q}-\varepsilon_{v}$ " paths, and shear characteristic $\left." q^{\prime}-\varepsilon_{q} "\right)$.
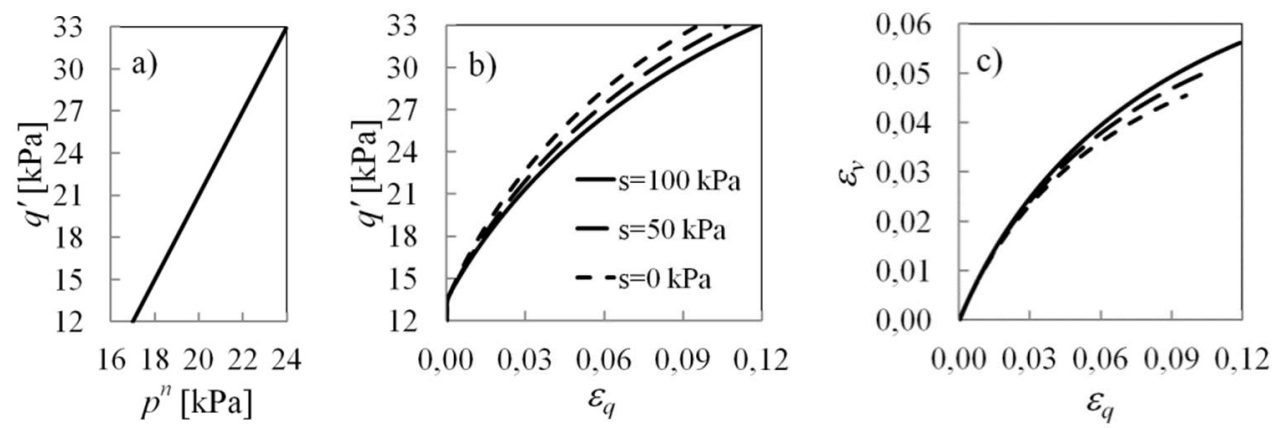

Fig. 3. Results: a) stress path " $q^{\prime}-p^{n "}$, b) shear characteristic " $q^{\prime}-\varepsilon_{q}$ ", c) strain path " $\varepsilon_{q}-\varepsilon_{v}$ ".

Their distribution shows that the increase in the suction level induces a significant increase of strain intensity $\varepsilon_{q}$ and volume strain $\varepsilon_{v}$ in the soil. In the analyzed case, their increase reaches over 10-20 percent in relation to the suction value equal to $0 \mathrm{kPa}$, which is a significant change with a small increase in the stress state in the numerical simulation of this stress path.

\section{Summary}

The presented method of numerical simulation of stress paths in the triaxial test, allows for an effective analysis of the suction influence on the behaviour of elasto-plastic models of the unsaturated soil medium, and verification of their ability to describe it properly. 


\section{References}

1. P. Fedczuk, AGH J. of Min. and Geoeng., 36, 2, 137-144 (2012)

2. P. Fedczuk, Eds J. Bzówka, M. Łupieżowiec, Analizy i doświadczenia w geoinżynierii, 99-106 (2017)

3. M. Gryczmański, Reologiczny model o wzmocnieniu anizotropowym dla szkieletu gruntowego, ZN WSI Opole, Bud., 20 (1980)

4. Y. Yu, K. Axelsson, Constitutive Driver for Response Behaviuor of Soils. Research Report (1992)

5. G.C. Nayak, O.C. Zienkiewicz, Int. J. for Num. Meth. in Eng., 5,113-135 (1972)

6. P. Fedczuk, Inż. i Bud., 11, 595-597 (2016)

7. P. Fedczuk, In: Budownictwo, ZN Polit. Biał., 28, 2, 83-92 (2006)

8. D.G. Fredlund, H. Rahardjo, Soil Mechanics for Unsaturated Soils (1993)

9. K.H. Roscoe, J.B. Burland, Eds J. Heyman, F.A. Leckie, Engineering Plasticity, 535608 (1968)

10. D. Sheng, S.W. Sloan, A. Gens, D.W. Smith, Int. J. for Num. and Anal. Meth. in Geomech., 27, 745-765 (2003)

11. D. Sheng, D.W. Smith, S.W. Sloan, A. Gens, Int. J. for Num. and Anal. Meth. in Geomech., 27, 767-790 (2003)

12. S.J. Wheeler, V. Sivakumar, Geotech., 45, 1, 35-53 (1995) 